\title{
Utility of Platelet Count for Predicting Cirrhosis in Alcoholic Liver Disease: Model for Identifying Cirrhosis in a US Population
}

\author{
Arvind R. Murali, MD ${ }^{7,3}$, Bashar M. Attar, MD, PhD'², Ariel Katz, MD ${ }^{7}$, Vikram Kotwal, $M D^{2}$, and Peter M. Clarke, MD \\ 'Department of Internal Medicine, John H Stroger Hospital of Cook County, Chicago, IL, USA; ${ }^{2}$ Department of Gastroenterology \& Hepatology, \\ John H Stroger Hospital of Cook County, Chicago, IL, USA; ' ${ }^{3}$ Department of Gastroenterology \& Hepatology, University of lowa Hospitals and Clinics, \\ lowa City, IA, USA.
}

\begin{abstract}
BACKGROUND: Thrombocytopenia has been shown to be the single most useful laboratory investigation for identifying subclinical cirrhosis of varying etiologies. However, alcohol per se can result in thrombocytopenia, and hence it is unclear whether platelet count can identify cirrhosis in patients who are alcoholic.
\end{abstract}

OBJECTIVES: To characterize the utility of clinical predictors, especially platelet count, for identifying the presence of cirrhosis in alcoholics. To develop a simple, objective model for identifying cirrhosis in alcoholics.

DESIGN: Retrospective cohort study.

PARTICIPANTS: A total of 2,471 consecutive hospitalized patients with abnormal liver enzyme levels were screened, from which 272 patients with a history of recent and ongoing alcohol intake, negative diagnostic studies for alternative etiologies of chronic liver disease, and recent liver imaging with ultrasound or CT scan were included.

MAIN MEASURES: Results of liver imaging and admission laboratory studies including liver enzymes, coagulation studies, and blood counts.

KEY RESULTS: One hundred twenty-nine patients (47\%) had cirrhosis based on imaging; 143 patients (53\%) had no cirrhosis. A pre-sobriety platelet count (during ongoing alcohol intake) of less than $70 * 10^{3}$ cells / $\mathrm{mm}^{3}$ was effective for ruling in cirrhosis (positive likelihood ratio [LR] 6.8, $95 \%$ CI: 3.4, 14); platelet count greater than $200^{*} 10^{3}$ was useful for ruling out cirrhosis in alcoholics (negative LR 0.18, 95 \% CI: 0.10, 0.35). Multivariate logistic regression analysis identified international normalized ratio (INR) $(p<0.01)$ and pre-sobriety platelet count $(p<0.01)$ as independent predictors of cirrhosis. A Model for identifying Cirrhosis in Alcoholic Liver Disease (MCALD) was developed using the INR and pre-sobriety platelet count; it had an area under the receiver operating characteristic curve of 0.89 and Hosmer-Lemeshow goodness of fit $\mathrm{chi}^{2}$ ( $p$ value) of $8.9(0.35)$ for predicting cirrhosis in alcoholics. A MCALD score $>5.5$ corresponded to an increased likelihood of cirrhosis (LR: 6.5, 95 \% CI: 4.3, 11.0) and a MCALD score $<5.5$ corresponded to decreased likelihood of cirrhosis in alcoholics (LR: 0.25, $95 \% \mathrm{CI}$ : $0.19,0.36$ ). Sobriety platelet count (after alcohol abstinence) at a cutoff of $160 * 10^{3}$ had positive LR of $7.9(95 \%$ CI: $4.4,14)$ and negative LR of 0.42 (95\% CI: 0.34, 0.52) for predicting cirrhosis in alcoholics.

Received May 14, 2014

Revised October 3, 2014

Accepted January 30, 2015

Published online February 21, 2015
CONCLUSIONS: A simple model of platelet count and INR has good diagnostic accuracy for identifying cirrhosis in alcoholics.

KEY WORDS: thrombocytopenia; alcoholism; cirrhosis; international normalized ratio; likelihood ratio.

J Gen Intern Med 30(8):1112-7

DOI: $10.1007 / \mathrm{s} 11606-015-3238-1$

(c) Society of General Internal Medicine 2015

\section{INTRODUCTION}

In the United States, cirrhosis is the 10th leading cause of death overall, with mortality rates of approximately 9.3 per 100,000 persons. The number of deaths from alcoholic liver disease in 2010 was reported to be $15,990^{1}$. Globally, in 2010 , alcoholattributable liver cirrhosis was responsible for 493,300 deaths and 14,544,000 disability-adjusted life years (DALYs), representing $0.9 \%$ ( $0.7 \%$ for women and $1.2 \%$ for men) of all deaths and $0.6 \%$ ( $0.4 \%$ for women and $0.8 \%$ for men $)$ of all DALYs worldwide ${ }^{2}$. If cirrhosis is identified early, interventions to prevent morbidity such as endoscopic screening for varices and hepatocellular carcinoma surveillance may influence outcome. Furthermore, advice for abstinence may be bolstered.

Aberrations in liver enzymes identify patients with alcoholic liver disease but cannot specifically sort those with cirrhosis from those with a less ominous type of liver disease. The diagnosis of cirrhosis is usually straightforward in a patient who has massive ascites, variceal hemorrhage, or hepatic encephalopathy. However, identifying cirrhosis in a patient with abnormal liver enzymes and previously unsuspected cirrhosis (subclinical cirrhosis) is challenging. Most patients with early cirrhosis have no clinical manifestations of the disease. Liver biopsy is the gold standard, but is invasive, and rare complications such as bleeding can be lethal ${ }^{3,4}$. Moreover, it is subject to sampling error and inter-observer variability. Ultrasonography identifies cirrhosis with reasonable sensitivity and specificity ${ }^{5}$, but may not be feasible in every case. Studies have shown that thrombocytopenia is the single most useful laboratory predictor of cirrhosis with viral etiology ${ }^{6}$. In cirrhosis, thrombocytopenia occurs as a result of a combination of hypersplenism secondary to portal hypertension and impaired thrombopoiesis from inadequate hepatic cell generation of thrombopoietin. However, in cirrhosis of 
alcoholic etiology, the utility of platelet count to help differentiate between cirrhotic and non-cirrhotic liver disease is unclear, as alcohol per se can cause thrombocytopenia by bone marrow depression and direct platelet toxicity ${ }^{7,8}$. It has been reported that regular administration of alcohol for 3 to 5 weeks causes an isolated fall in platelet count ${ }^{7}$. This effect of alcoholinduced thrombocytopenia lasts for 5 to 7 days after alcohol abstinence $^{7}$, and the platelet count should reach its normal level after 7 days of abstinence from alcohol intake. Various prediction models and scoring systems have been proposed for predicting cirrhosis in alcoholics, but these models have either not been validated or were found to be too specialized and not routinely available for clinical use ${ }^{9-11}$. Therefore, we aimed to study the role of platelet count for identifying underlying cirrhosis in patients with alcoholic liver disease and with recent alcohol intake. We also aimed to develop and test a simple, objective model for predicting cirrhosis in alcoholics.

\section{METHODS}

\section{Study population}

We screened 2,471 consecutive patients with abnormal liver enzymes admitted to the John H. Stroger Jr. Hospital of Cook County, Chicago, IL, USA, from January 2009 through December 2011. We used electronic medical records to select those with a negative hepatitis profile and who had an abdominal ultrasound or computed tomography (CT) performed within 3 months of admission. Among these, only patients with documentation of recent and ongoing alcohol intake (greater than $30 \mathrm{~g}$ /day for a period of at least 3 weeks prior to admission, with the last drink within 5 days of laboratory tests) and no evidence of any other etiology for elevated liver enzymes were included in the study. We excluded patients who had positive antinuclear antibody, anti-smooth muscle antibody, anti-mitochondrial antibody, and in whom liver biopsy suggested or confirmed an alternative etiology for elevated liver enzymes such as hemochromatosis, autoimmune hepatitis, or non-alcoholic steatohepatitis. We also excluded patients who had an alternate condition that could cause thrombocytopenia such as sepsis, autoimmune diseases such as lupus, and hematologic or oncologic malignancy. Patients who had clinically obvious ascites, who presented with variceal hemorrhage or overt hepatic encephalopathy, and patients who had a past history of ascites or esophageal varices were excluded from the study. Patients who did not have a liver ultrasound or an abdominal computed tomography were excluded as well, as the outcome of cirrhosis was measured based on imaging studies. The patients included in our study were admitted to the general internal medicine inpatient units for evaluation and management of alcohol intoxication or alcohol withdrawal or possible alcoholic hepatitis. Patients who were not actively drinking were excluded from the study. Data was extracted by one investigator (ARM) and verified by another investigator (VK).

\section{Measurements}

We recorded demographic data such as age and gender. We collected and analyzed the admission laboratory variables, namely, serum sodium, serum creatinine, total protein, serum albumin, total bilirubin, direct bilirubin, alkaline phosphatase, gamma-glutamyl transferase (GGT), aspartate transaminase (AST), alanine transaminase (ALT), lactate dehydrogenase, total cholesterol, white cell count, platelet count, and international normalized ratio (INR). We also calculated and recorded the Model for End-Stage Liver Disease (MELD) score and the AST to platelet count ratio index (APRI) based on the admission variables. The platelet count that was obtained at the time of admission was the platelet count during the phase of active alcohol intake, and hence we named this the 'pre-sobriety'

Table 1 Bivariate analyses of demographic and laboratory variables in identifying cirrhosis in alcoholics

\begin{tabular}{|c|c|c|c|}
\hline Variable & $\begin{array}{l}\text { No Cirrhosis mean }(95 \% \text { CI }) \\
N=143\end{array}$ & $\begin{array}{l}\text { Cirrhosis mean (95 \% CI) } \\
N=129\end{array}$ & P value \\
\hline Age & $47.9(46.3,49.4)$ & $50.4(48.6,52.1)$ & 0.04 \\
\hline Serum sodium $(\mathrm{mEg} / \mathrm{L})$ & $134.7(133.9,135.6)$ & $134.9(134,135.8)$ & 0.83 \\
\hline *Serum creatinine $(\mathrm{mg} / \mathrm{dl})$ & $0.8(0.4,7.7)$ & $0.9(0.4,7.2)$ & 0.83 \\
\hline Total protein $(\mathrm{g} / \mathrm{dl})$ & $6.83(6.7,7)$ & $6.77(6.6,6.9)$ & 0.63 \\
\hline Serum albumin $(\mathrm{g} / \mathrm{dl})$ & $3.64(3.5,3.8)$ & $2.9(2.8,3)$ & $<0.01$ \\
\hline *Total bilirubin (mg/dl) & $4.9(0.2,42.7)$ & $7.0(0.5,61)$ & 0.07 \\
\hline $\mathrm{ALP}(\mathrm{IU} / \mathrm{L})$ & $171.7(142.7,200)$ & $182.4(158.4,206.3)$ & 0.57 \\
\hline *GGT (IU/L) & $364(30,5667)$ & $340(29,3010)$ & 0.09 \\
\hline AST (IU/L) & $167.8(140.6,194.9)$ & $139.8(115.7,163.9)$ & 0.13 \\
\hline ALT (IU/L) & $89.7(75.4,104)$ & $65.9(49.8,82.1)$ & 0.03 \\
\hline LDH (IU/L) & $316.8(285.2,348.4)$ & $305.2(276.6,333.8)$ & 0.59 \\
\hline Total cholesterol $(\mathrm{mg} / \mathrm{dl})$ & $221.8(200,243.5)$ & $137.8(123.7,152)$ & $<0.01$ \\
\hline Pre-sobriety platelet count (cells $/ \mathrm{mm}^{3} * 10^{3}$ ) & $189.8(171.8,207.8)$ & $111.4(99.4,123.4)$ & $<0.01$ \\
\hline Sobriety platelet count (cells $\left./ \mathrm{mm}^{3} * 10^{3}\right)$ & $264.4(242.1,287)$ & $99.1(85.9,112)$ & $<0.01$ \\
\hline INR & $1.1(1.0,1.2)$ & $1.6(1.5,1.7)$ & $<0.01$ \\
\hline MELD score & $8.4(7.1,9.8)$ & $15.5(13.6,17.4)$ & $<0.01$ \\
\hline APRI index & $122.5(98.6,146.4)$ & $190.8(146.2,235)$ & 0.01 \\
\hline
\end{tabular}

Bivariate analysis performed using $t$ test. ${ }^{*}$ indicates median (ranges) reported for non-normal variables. CI confidence interval, ALP alkaline phosphatase, GGT gamma-glutamyl transpeptidase, AST aspartate transaminase, ALT alanine transaminase, LDH lactate dehydrogenase, INR international normalized ratio, MELD Model for End-Stage Liver Disease, APRI AST to Platelet count ratio index 
platelet count. Whenever available, we also recorded the platelet count obtained after a minimum of 7 days of alcohol abstinence. We labeled this platelet count the 'sobriety' platelet count. We chose a minimum period of 7 days of alcohol abstinence, as the effect of alcohol-induced thrombocytopenia is expected to last 5-7 days ${ }^{7}$. Cirrhosis was the outcome of interest in our study. The presence or absence of cirrhosis was determined solely on the basis of imaging studies.

\section{Statistical analysis}

We estimated based on prior studies that the sensitivity and specificity of platelet count at a cutoff of $160 * 10^{3}$ cells $/ \mathrm{mm}^{3}$ as a diagnostic test to predict cirrhosis would be about $70 \%$ and $80 \%$, respectively. Assuming that a clinically meaningful upper limit for the negative likelihood ratio was 0.5 , we calculated a sample size of 110 patients in each arm, assuming an equal ratio of cirrhosis and no cirrhosis ${ }^{12}$.

Continuous variables were tested for normal distribution and were expressed as mean (standard deviation), whereas non-continuous variables were presented as medians and ranges. Bivariate analysis was performed using $t$ tests, with cirrhosis as the outcome of interest to compare the two groups.

Univariate logistic regression analysis was performed to identify significant variables in the identification of cirrhosis. Covariates with a $\mathrm{P}$ value of 0.10 or less were considered significant. A p value of 0.10 was used in order to avoid missing significant variables. The variables found to be significant in the univariate analysis, as well as variables thought to be clinically significant, were all included in the multivariate analysis. Logistic regression analysis with a stepwise backward elimination approach was used for model development. Predictive accuracy and calibration of the models were compared using receiver operating characteristic (ROC) curves and the HosmerLemeshow goodness of fit test. Bootstrapping, a re-sampling technique, was performed to validate the model in our population. Random sampling with replacement was performed, and 1,000 samples were obtained.

Table 2 Multivariate logistic regression analysis of variables obtained at the time of active alcohol intake in identifying cirrhosis in alcoholics

\begin{tabular}{llc}
\hline \hline Variable & OR (95 \% CI) & P value \\
\hline Age & $1.01(0.97,1.05)$ & 0.49 \\
Serum albumin (g/dl) & $0.59(0.31,1.11)$ & 0.10 \\
Total bilirubin (mg/dl) & $0.98(0.91,1.06)$ & 0.78 \\
GGT (IU/L) & $1.00(0.99,1.00)$ & 0.75 \\
ALT (IU/L) & $1.00(0.99,1.00)$ & 0.57 \\
Total cholesterol (mg/dl) & $0.99(0.99,1.00)$ & 0.45 \\
Pre-sobriety platelet count & $0.987(0.97,0.99)$ & $<0.01$ \\
(cells/mm $\left.{ }^{3} * 1^{3}\right)$ & $278.51(28.29,2741.36)$ & $<0.01$ \\
INR & $0.95(0.87,1.03)$ & 0.28 \\
MELD score & $0.99(0.99,1.00)$ & 0.60 \\
APRI index &
\end{tabular}

OR odds ratio, CI confidence interval, ALP alkaline phosphatase, GGT gamma-glutamyl transpeptidase, AST aspartate transaminase, ALT alanine transaminase, $L D H$ lactate dehydrogenase, INR international normalized ratio, MELD Model for End-Stage Liver Disease, APRI AST to platelet count ratio index
Table 3 MCALD model developed by logistic regression analysis

\begin{tabular}{llll}
\hline \hline Variable & $\begin{array}{l}\text { Coefficient } \\
(\mathbf{9 5} \% \mathbf{C I})\end{array}$ & $\begin{array}{l}\text { Standard } \\
\text { error }\end{array}$ & $\begin{array}{l}\text { P } \\
\text { value }\end{array}$ \\
\hline INR & $5.3(3.74,6.71)$ & 0.756 & $<0.01$ \\
Pre-sobriety platelet & $-0.0106(-0.015$, & 0.002 & $<0.01$ \\
count & $-0.006)$ & & \\
\hline
\end{tabular}

INR international normalized ratio, CI confidence interval

A risk score formula to predict cirrhosis was developed using variables significant on multivariate analysis. A p value of less than 0.05 was considered significant on multivariate analysis. A logistic regression model was used to develop the weights needed to create the risk score formula. The regression coefficients were exponentiated and then used to create the risk score. The sensitivity and specificity at various cutoff points of the risk score was calculated, and the score with the best sensitivity without compromising the specificity and vice versa, was chosen as the best cutoff value.

The sensitivity, specificity, positive and negative likelihood ratios for the various cutoffs and ranges of the platelet count and for the newly developed risk score were calculated and recorded. All analyses were performed using Stata 9.1 statistical software, TX, USA.

The study was approved by the institutional review board of John H. Stroger Jr. Cook County Hospital.

\section{RESULTS}

Of the 2,471 patients in the database from 2009 to 2011 with elevated liver enzymes, 2,199 patients were excluded from the study. Reasons for exclusion were absence of documentation of recent alcohol intake, presence of an additional or alternate etiology for abnormal liver enzymes, and the presence of a coexisting condition capable of causing thrombocytopenia. A total of 272 patients satisfied our inclusion criteria. Of these, $129(47 \%)$ had cirrhosis. The majority of the patients were

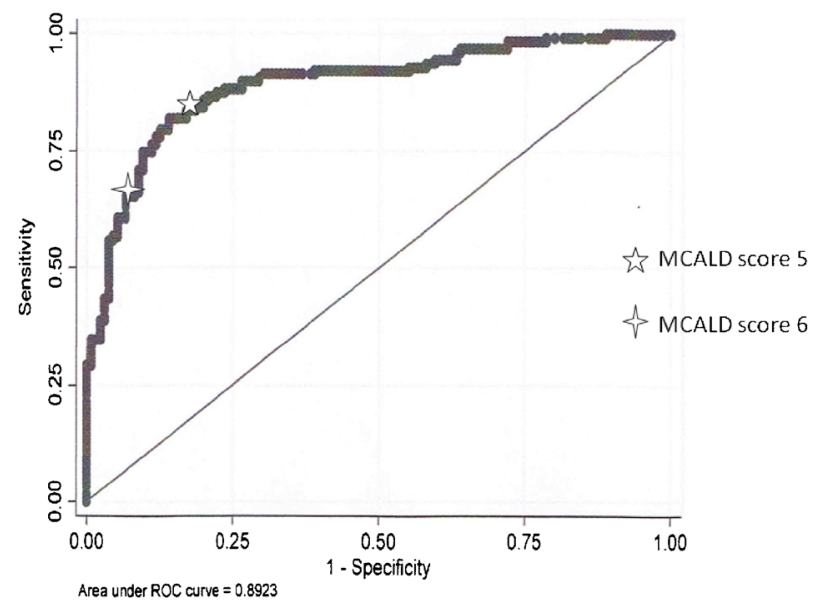

Fig. 1 Area under the receiver operating characteristic curve of MCALD model to identify cirrhosis in alcoholics 
Table 4 Likelihood ratios of various cutoffs of pre-sobriety platelet count to identify cirrhosis in alcoholics

\begin{tabular}{|c|c|c|c|c|c|c|c|c|}
\hline $\begin{array}{l}\text { Platelet count } \\
(10 * 3)\end{array}$ & $\begin{array}{l}\text { No of } \\
\text { patients (n) }\end{array}$ & $\begin{array}{l}\text { Patients with } \\
\text { cirrhosis }\end{array}$ & Sensitivity & Specificity & $\begin{array}{l}\text { Positive LR } \\
\text { (95\% CI) }\end{array}$ & $P$ value & $\begin{array}{l}\text { Negative LR } \\
(95 \% \text { CI })\end{array}$ & P value \\
\hline$<70$ & 51 & 44 & 0.34 & 0.95 & $6.8(3.4,14)$ & $<0.01$ & $0.69(0.6,0.82)$ & $<0.01$ \\
\hline$<100$ & 85 & 64 & 0.50 & 0.85 & $3.3(2.2,4.9)$ & $<0.01$ & $0.59(0.5,0.74)$ & $<0.01$ \\
\hline$<130$ & 124 & 85 & 0.66 & 0.73 & $2.4(1.8,3.2)$ & $<0.01$ & $0.47(0.34,0.64)$ & $<0.01$ \\
\hline$<160$ & 168 & 103 & 0.80 & 0.55 & $1.8(1.5,2.2)$ & $<0.01$ & $0.36(0.23,0.57)$ & $<0.01$ \\
\hline
\end{tabular}

LR likelihood ratio

male (76\%). The distinction in clinical and laboratory variables between patients with and without cirrhosis is given in Table 1 .

Univariate logistic regression analysis of admission variables identified age $(p=0.04)$, serum albumin $(p<0.01)$, serum total cholesterol $(p<0.01)$, pre-sobriety (admission) platelet count $(p<0.01)$, INR $(p<0.01)$, and MELD score $(p<0.01)$ as significant in predicting cirrhosis.

Multivariate logistic regression analysis of admission variables identified only pre-sobriety platelet count $(P<0.01)$ and INR $(P<0.01)$ as significant independent predictors of cirrhosis (Table 2). A Model for identifying Cirrhosis in Alcoholic Liver Disease (MCALD) was developed using only two objective variables: pre-sobriety platelet count and INR (Table 3). The predictive accuracy of MCALD in predicting cirrhosis in patients with alcoholic liver disease was $0.89(P<0.01 ; 95 \%$ CI: $0.85,0.92$ ) (Fig. 1). The model calibrated well with a Hosmer-Lemeshow goodness of fit $\mathrm{chi}^{2}$ of 8.9 and $P=0.35$. Addition of other variables did not improve the model.

The formula for calculating the MCALD score is: 5.3*INR0.01*platelet count. The cutoff of the MCALD score that jointly maximizes the sensitivity and specificity to identify cirrhosis is 5.5 , with a sensitivity of $78 \%$, specificity of $88 \%$, positive likelihood ratio (LR) of 6.5 (95\% CI: 4.3, 11.0), and negative likelihood ratio of 0.25 (95\% CI: 0.19 , $0.36)$.

Bootstrapping of the MCALD model showed a 0.122 bias in the beta weight in the INR estimate, and 0.0004 bias in beta weight in the pre-sobriety platelet count estimate, indicating that the model may perform well in a clinical setting in the population studied.

\section{Likelihood ratios}

The mean pre-sobriety platelet count in patients without cirrhosis was $189.8^{*} 10^{3}$ cells $/ \mathrm{mm}^{3}$ and in patients with cirrhosis

Table 5 Likelihood ratios of various ranges of pre-sobriety platelet count to identify cirrhosis in alcoholics

\begin{tabular}{llll}
\hline \hline Platelet count & $\begin{array}{l}\text { No of } \\
\text { patients }\end{array}$ & $\begin{array}{l}\text { Patients with } \\
\text { cirrhosis (\%) }\end{array}$ & LR (95 \% CI) \\
\hline$<70$ & $\mathbf{5 1}$ & $\mathbf{4 4}(\mathbf{8 6 . 2 )}$ & $\mathbf{6 . 8}(\mathbf{3 . 4}, \mathbf{1 4})$ \\
$70-99$ & 34 & $20(58.8)$ & $1.6(0.83,3.0)$ \\
$100-129$ & 39 & $21(53.8)$ & $1.3(0.7,2.3)$ \\
$130-159$ & 34 & $18(52.9)$ & $1.2(0.7,2.3)$ \\
$160-199$ & 45 & $16(36)$ & $0.7(0.4,1.1)$ \\
$>\mathbf{2 0 0}$ & $\mathbf{6 9}$ & $\mathbf{1 0}(\mathbf{1 4 . 5 )}$ & $\mathbf{0 . 1 8}(\mathbf{0 . 1 0}, \mathbf{0 . 3 5})$ \\
\hline
\end{tabular}

LR likelihood ratio was $111.4 * 10^{3}$ cells $/ \mathrm{mm}^{3}$ ( $p$ value $<0.01$ ). Tables 4 and 5 provides the sensitivity, specificity, and positive and negative LR at different cutoffs and ranges of pre-sobriety platelet count to predict alcoholic cirrhosis.

The sensitivity, specificity, and positive and negative LR for various ranges of the MCALD score to predict alcoholic cirrhosis are provided in Tables 6 and 7.

\section{Platelet count after minimum of 7 days of alcohol abstinence (sobriety platelet count)}

The mean sobriety platelet count in patients without cirrhosis was $264.4^{*} 10^{3}$ cells $/ \mathrm{mm}^{3}$ and in patients with cirrhosis was $99.1 * 10^{3}$ cells $/ \mathrm{mm}^{3}(p<0.01)$. Univariate analysis identified sobriety platelet count as a significant predictor of cirrhosis (Table 1). A sobriety platelet count cutoff of $160 * 10^{3}$ had a sensitivity of $61 \%$, specificity of $92 \%$, positive LR of 7.9 (CI: $4.4,14)$, and negative LR of 0.42 (CI: $0.34,0.52$ ) for identifying cirrhosis in alcoholics.

\section{DISCUSSION}

Patients with abnormal liver enzyme levels as a result of alcoholic liver disease are frequently encountered by both general physicians and specialists. Identifying cirrhosis in these patients is of the utmost importance. Liver biopsy is untenable as a surveillance method for all patients at risk.

Numerous non-invasive serologic markers have been proposed for diagnosing alcoholic cirrhosis. The AST platelet count index (APRI index) that was developed was shown to have good sensitivity and specificity for predicting cirrhosis in patients with hepatitis $\mathrm{C}$, but in patients with alcoholic cirrhosis it was found to have low sensitivity and specificity ${ }^{10}$. The PGA index (prothrombin, gamma glutamyl transpeptidase, and apolipoprotein AI [ApoA1] levels) has been shown to have good accuracy for detecting cirrhosis in patients with alcoholic liver disease ${ }^{11}$. The FibroIndex uses the platelet count, AST level, and gamma globulin level to detect significant fibrosis in chronic hepatitis $\mathrm{C}^{12}$, but its accuracy has yet to be validated. FibroTest and FibroSure are commercial tests for predicting fibrosis using the levels of alpha-2 macroglobulin, alpha-2 globulin, gamma globulin, apolipoprotein AI, gamma-glutamyl transferase, and total bilirubin, and they have been reported to have decent sensitivity and specificity in identifying cirrhosis. The FibroScan, or transient elastography, which was developed to allow evaluation of liver fibrosis by 
Table 6 Likelihood ratios at various cutoffs of MCALD score to identify cirrhosis in alcoholics

\begin{tabular}{|c|c|c|c|c|c|c|c|c|}
\hline $\begin{array}{l}\text { MCALD } \\
\text { score }\end{array}$ & $\begin{array}{l}\text { No of } \\
\text { patients }\end{array}$ & $\begin{array}{l}\text { Patients with } \\
\text { cirrhosis }\end{array}$ & Sensitivity & Specificity & $\begin{array}{l}\text { Positive LR } \\
\text { (95\%CI) }\end{array}$ & P value & $\begin{array}{l}\text { Negative LR } \\
\text { (95\% CI) }\end{array}$ & P value \\
\hline$>5$ & 133 & 107 & 0.83 & 0.81 & $4.5(3.1,6.5)$ & $<0.01$ & $0.21(0.14,0.31)$ & $<0.01$ \\
\hline$>6$ & 97 & 85 & 0.67 & 0.91 & $7.9(4.6,14)$ & $<0.01$ & $0.36(0.28,0.46)$ & $<0.01$ \\
\hline$>7$ & 70 & 65 & 0.50 & 0.97 & $14.4(6,34)$ & $<0.01$ & $0.51(0.43,0.61)$ & $<0.01$ \\
\hline
\end{tabular}

$* M C A L D$ score $=5.3 * I N R-0.01 *$ platelet count, LR likelihood ratio

measuring liver stiffness, has shown promising results ${ }^{13}$. The major problem with these models is that the components of the tests are not readily available in most clinical laboratories; some of the tests are expensive, and others are too cumbersome to be of use to the physician in a clinical setting. Thus there is a need to develop a simple, objective model to help identify cirrhosis in alcoholics.

A recent meta-analysis by Udell et al. ${ }^{6}$ identified thrombocytopenia as the single most important laboratory test for identifying cirrhosis of varying etiologies. The authors concluded that a platelet count cutoff of $160 * 10^{3}$ has a positive LR of 6.3 and negative LR of 0.29 in predicting cirrhosis of varying etiologies. However, almost all of the studies used in the meta-analyses were performed on patients with cirrhosis of viral etiology.

In our study, we evaluated patients along the spectrum of alcoholic liver disease and who were recent alcoholics. With regard to the pre-sobriety (admission) platelet count, we found that a platelet count cu-off of $160 * 10^{3}$ cells/ $\mathrm{mm}^{3}$ had a poor LR for predicting cirrhosis in alcoholics. This is likely explained by the direct toxic effect of alcohol on platelet count ${ }^{8}$. We found, however, that a pre-sobriety platelet count of less than $70^{*} 10^{3}$ was effective for ruling in alcoholic cirrhosis $(\mathrm{LR}+6.8)$, and a platelet count greater than $200 * 10^{3}$ significantly decreased the likelihood of having cirrhosis (LR- 0.18), which is of clinical value. A pre-sobriety platelet count ranging from $70^{*} 10^{3}$ to $200^{*} 10^{3}$ was ineffective for ruling in or ruling out cirrhosis. Unfortunately, $56 \%$ of the patients in our study had a platelet count within this range, and hence pre-sobriety platelet count alone is not sufficient for identifying cirrhosis in this patient group.

With the addition of the INR to the pre-sobriety platelet count, the MCALD model had good diagnostic ability in identifying cirrhosis in alcoholics, as shown by the area under

Table 7 Likelihood ratios of various ranges of MCALD score to identify cirrhosis in alcoholics

\begin{tabular}{llll}
\hline \hline MCALD score & $\begin{array}{l}\text { No of } \\
\text { patients }\end{array}$ & $\begin{array}{l}\text { Patients with } \\
\text { cirrhosis (\%) }\end{array}$ & $\begin{array}{l}\text { LR } \\
\mathbf{( 9 5} \% \mathbf{C I})\end{array}$ \\
\hline$<4$ & 84 & $12(14.2)$ & $0.18(0.1,0.3)$ \\
$4-5$ & 55 & $10(18.2)$ & $0.25(0.1,0.4)$ \\
$5-6$ & 36 & $22(61.1)$ & $1.7(0.9,3.2)$ \\
$6-7$ & 27 & $20(74)$ & $3.2(1.4,7.2)$ \\
$>7$ & 70 & $65(92.8)$ & $14.4(6,34)$ \\
\hline
\end{tabular}

$*$ MCALD score $=5.3 * I N R-0.01 *$ platelet count, LR likelihood ratio the ROC of 0.89. A MCALD score greater than 6 was highly suggestive of cirrhosis, with LR of 7.9 (95\% CI: 4.6, 14), while a score of less than 5 was effective for indicating a reduced likelihood of cirrhosis, with LR of 0.21 (95\% CI: $0.14,0.31$ ). Only $13 \%$ of patients had non-diagnostic MCAL D scores from 5 to 6 .

A platelet count after a minimum of 7 days of alcohol abstinence (the 'sobriety' platelet count) was found to be useful for identifying cirrhosis in alcoholics. It performed better than the pre-sobriety platelet count alone in differentiating alcoholic patients with cirrhosis from patients without cirrhosis. In comparing the pre-sobriety platelet count to the sobriety platelet count, we note that the sobriety platelet count at a cutoff of $160 * 10^{3}$ had a positive LR of 7.9 (CI: 4.4, 14) while the pre-sobriety platelet count at a cutoff of $160 * 10^{3}$ had a positive LR of just 1.8 $(1.5,2.2)$ for identifying cirrhosis in alcoholics. This superiority of the sobriety platelet count over the presobriety platelet count was consistent at various cutoffs of the platelet count. This difference in diagnostic ability is due to the direct toxic effect of alcohol on the presobriety platelet count, which is no longer evident on the platelet count obtained after a minimum of 7 days of alcohol abstinence. As such, the platelet count obtained after a period of alcohol abstinence at a cutoff of $160^{*} 10^{3}$ cells $/ \mathrm{mm}^{3}$ had a likelihood ratio similar to that of the platelet count in the study published by Udell et al. ${ }^{6}$, suggesting that after a minimum 7 days of alcohol abstinence, the diagnostic utility of platelet count in alcoholic cirrhosis was similar to that in cirrhosis of viral etiology. However, it may not be possible to obtain a sobriety platelet count in all alcoholic patients.

The major limitation in our study was the use of imaging to diagnose cirrhosis rather than the gold standard of liver biopsy. However, in the current era, liver biopsy is rarely used to diagnose alcoholic cirrhosis, and in a majority of cases, the diagnosis is made by imaging studies. Another limitation of our study was its retrospective design. To overcome this limitation, we estimated the sample size needed to power our study and also provided the confidence intervals for all of our likelihood ratios. We could not be sure why the excluded patients did not receive imaging, and this may have skewed our sample. This was offset, however, by the large number of patients and relative uniformity of care. We did not study the correlation between the quantities of alcohol intake with the level of thrombocytopenia. Although patient reporting of 
alcohol intake may be a limitation from an efficacy perspective, it is similar to the history that would generally be obtained in real life, and hence may be a strength from an effectiveness perspective. Finally, some of the patients in our study may have been taking over-the-counter medications such as antiinflammatory agents or antidepressants, which may have contributed to thrombocytopenia.

\section{CONCLUSIONS}

A simple model consisting of two objective variables - namely, pre-sobriety platelet count and INR - is reliable for identifying cirrhosis in patients with alcoholic liver disease with recent and ongoing alcohol intake. A platelet count obtained after a minimum of 7 days of alcohol abstinence is also effective for differentiating alcoholic patients with cirrhosis from those without cirrhosis. Further prospective studies across other institutions are needed to evaluate the MCALD model.

Acknowledgments: 1) William Trick, MD. Department of Internal Medicine, Collaborative Research Unit, John H. Stroger Hospital of Cook County, Chicago, IL, USA

2) Helen Zhang, BS: Data Analyst, Collaborative Research Unit, John H. Stroger Hospital of Cook County, Chicago, IL, USA

There was no funding associated with this project.

No contributors to this project.

No prior presentations.

Conflict of Interest: The authors declare that they have no conflicts of interest.
Corresponding Author: Arvind R. Murali, MD; Department of Internal MedicineJohn H Stroger Hospital of Cook County, Chicago, IL, USA (e-mail: arvindrm13@gmail.com).

\section{REFERENCES}

1. Rehm J, Samokhvalov AV, Shield KD. Global burden of alcoholic liver diseases. J Hepatol. 2013;59(1):160-8. doi:10.1016/j.jhep.2013.03.007.

2. Hoyert, Xu. National Vital Statistics Reports, Vol. 61, No. 6, October 10, 2012

3. Rockey X, Caldwel X, Goodman X, Nelson X, Smith X. Liver Biopsy. American association for the study of liver diseases. Hepatology. 2009;49:1017-44.

4. Piccinino F, Sagnelli E, Pasquale G, Giusti G. Complications following percutaneous liver biopsy- A multicentre retrospective study on 68,276 biopsies. J Hepatol. 1986;2(2): 165-73.

5. Simonovski V. The diagnosis of cirrhosis by high resolution ultrasound of the liver surface. Br J Radiol. 1999;72(853):29-34.

6. Udell JA, Wang CS, Tinmouth J, et al. Does this patient with liver disease have cirrhosis? JAMA. 2012;307(8):832-42.

7. Handin, Lux, Stossel, et al. Blood: Principles and Practice of Hematology. Chapter 32: Platelet life cycle: Quantitative disorders. Page 1023.

8. Lindenbaum, Hargrove. Thrombocytopenia in Alcoholics. Ann Intern Med. 1968;68(3):526-32.

9. Lieber CS, Weiss DG, Morgan TR, Paronetto F. Aspartate aminotransferase to platelet ratio index in patients with alcoholic liver fibrosis. Am J Gastroenterol. 2006;101(7): 1500-8.

10. Poynard T, Aubert A, Bedossa P, et al. A simple biological index for detection of alcoholic liver disease in drinkers. Gastroenterology. 1991;100(5 Pt 1):1397-402.

11. Koda M, Matunaga Y, Kawakami M, Kishimoto Y, Suou T, Murawaki Y. FibroIndex, a practical index for predicting significant fibrosis in patients with chronic hepatitis C. Hepatology. 2007;45(2):297-306.

12. Simel DL, Samsa GP, Matchar DB. Likelihood ratios with confidence: sample size estimation for diagnostic test studies. J Clin Epidemiol. 1991;44(8):763-70.

13. Foucher J, Chanteloup E, Vergniol J, et al. Diagnosis of cirrhosis by transient elastography (FibroScan): a prospective study. Gut. 2006;55(3):403-8. 